\title{
Problems experienced by role players within the managed healthcare context in Gauteng
}

\author{
S Mahlo \\ M.Cur \\ RAU (masters student)
}

$\&$

M Muller

D. Cur.

Professor: Department of Nursing Science

RAU

\section{"Managed healtheare is a system of healtheare delivery that ensures that cost effective quality care is provided to patients, who are members of medical aid schemes within the managed healtheare context."}

\begin{abstract}
Role players within the context of managed healthcare in Gauteng experience problems in the delivery of healthcare, which negatively affect their working relationships. This in turn, affects the quality of care provided to patients. The purpose of this study is to explore and describe the problem experienced by different role players within the context of managed healthcare in Gauteng, as well as the suggested solutions to counteract these problems. These results will be utilised as the basis of a conceptual framework to formulate a strategy to enhance the working relationships amongst these role players. The strategy will not be discussed in this article as the focus is on the problems experienced by the role players in the delivery of healthcare, as well as suggested solutions in the counteraction thereof. A qualitative, exploratory, descriptive and contextual study was followed to explore and describe the problems, as well as the suggested solutions to counteract these problems. Focus group interviews were conducted to collect data from three private hospitals, three managed care organisations and four general medical practitioners in Gauteng. The participants were purposively and conveniently selected. Content analysis as described by Tesch (1990) was followed to analyse the data. The main problems experienced were related to inadequate communication, inadequate staff competence, cost saving versus quality care, procedural complexity, perceived loss of power by doctors and patients and the system of accounts payment. The suggested solutions focused mainly on empowerment and standardisation of procedures. It is recommended that replication studies of this nature be conducted in other provinces and that ethical standards are formulated within the managed healthcare context.
\end{abstract}

\section{Introduction}

Managed healthcare is a system of healthcare delivery that ensures that cost effective quality care is provided to patients, who are members of medical aid schemes within the managed healthcare context. Various role players are involved in the delivery of healthcare within the managed healthcare context. According to Anderson (in HASA 1998:170). there are three main role players in managed healthcare, namely; - the professional service providers, the funders and the members. Professional service providers are responsible for the provision of quality healthcare to patients. Funders, represented by managed care organisations, are responsible for paying for the healthcare services provided to patients by the professional service providers and to monitor the quality of care provided. In this context, professional service providers are representeeđ by the privaté hospitals and the doctors providing private healtcare services within the managed healthcare context in Gauteng. Members are responsible for the pay: ment of their monthly premiums to their managed care organisations. Medical aid schemes in South Africa, representing 15 to $20 \%$ of all patients (HASA, 1998:160), started introducing managed healthcare in an attempt to reduce healthcare costs, which was increasing at a high rate for the past few years, particularly so in the private healthcare industry.

According to T.W.I.G Inform (1998:6), managed healthcare was introduced four years ago in South Africa, and since then, an estimated $95 \%$ of local medical aids have introduced a form of cost savings orientated to the philosophy of managed healthcare. During the process of healthcare delivery, role players experience problems which negatively affects their working relationships and in turn, affect the quality of care provided to patients (Barr, 1997:4; Gallows and Lee, 1997:246). As managed healthcare is fairly new in South Africa, little is known about these problems. As a result, there 
are no guidelines for the formulation of a strategy to enhance positive working relationships among the role players. Exploration and description of the problems experienced by the role players and the suggested solutions to counteract these problems, within the context of managed healthcare, should provide guidelines for the researcher to formulate a strategy to enhance positive working relationships among the role players in the delivery of healthcare. This in turn, should impact upon the quality of care provided to patients. The study focuses on the problems experienced by the professional service providers and the funders. The following research question is relevant: "What are the problems experienced by the role players within the managed healthcare context in Gauteng and how can these problems be solved? The specific objective of the study is to explore and describe the problems experienced by the role players and the suggested solutions to counteract these problems, within the context of managed healthcare in Gauteng.

These results will form the basis for the development of the strategy to enhance positive working relationship among the role players within the context of managed healthcare in Gauteng.

\section{Terminology Managed healthcare}

Managed healthcare is a system of healthcare delivery that influences the utilisation and cost of services to facilitate cost effective quality care to the member of the medical aid scheme within the managed healthcare context.

\section{Role players}

Role players in this study refer to the professional service providers and the funders in the context of managed healthcare in Gauteng.

\section{Nursing service manager}

The nursing service manager is a registered nurse/midwife in charge of a nurs. ing service and is responsible for the facilitation of quality nursing care in a cost effective manner within the managed healthcare context.

\section{Init manager}

A unit manager is a registered nurse/ midwife in charge of a clinical medical ward/unit within the managed healthcare context where he/she acts as the operational manager of nursing care.

\section{Hospital case manager}

Hospital case manager refers to a registered nurse employed by the private hospital to liase with the managed care organisations and ensures that the hospital is getting paid for the services rendered to the patients, who are members of the managed care organisations.

\section{Managed care case managers}

Managed care case managers refer to registered nurses employed by managed care organisations to monitor the quality of care provided to their members by the service providers and to approve payment for such services. Managed care case managers ensure that professional service providers provide cost effective quality care to their members.

\section{Problems}

Problems refer to negative experiences by the role players within the managed healthcare context in the delivery of healthcare.

\section{Solutions}

The suggested strategies to counteract the problem.

\section{Research Design}

A qualitative, exploratory, descriptive and. confextual design was followed to explore the problems experienced by the role players within the context of managed healthcare in Gauteng, as well as the suggested solutions to counteract these problems. Focus group interviews were conducted to explore and describe the problems and the solutions to coun teract these problems (Krueger, 1988). Focus groups were conducted with three private hospitals, three managed care organisations and four general medical practitioners in Gauteng, All role players and participants were purposively and convententty selected according to the set inclusion criteria:

- The hospital had a medical unit/ward, nursing service manager and a hospital case manager.

- A nursing service manager with one year experience as a nursing service manager in a private hospital.

- Medical ward unit manager to have at least one year experience as a medical ward unit manager in a private hospital

- Hospital case manager with at least one year experience as a case manager.

- Managed care organisation must be in existence for a year.

- Managed care case managers with one year experience working as a managed care case manager

- Doctors must have been in private practice for at least a year.

The researcher used the principles of focus group interviews (Krueger, 1988) to encourage the participants to actively participate. The following principles are relevant:

- Only information rich participants who are willing to participant were selected

- The researcher used a group small enough to allow every member to participate and big enough to generate information

- Although they had common background, participants were not familiar with each other as this could influence the information they generate

- Focus group interviews took place in a place designed for the focus group interviews

- Discussion took place in a series to allow all participants to actively participate.

The following research question was asked: "What are the problems experienced by the role players within the context of managed healthcare in Gauteng and what solutions do you suggest to counteract these problems?". To facilitate data storage and retrieval, the re searcher opened three (De Vos, 1998:334). Comprehensive field notes were also written by the researcher to capture external factors that could have influenced the information generated and the researcher's experiences. Content analysis as suggested by Tesch (1990) was conducted and an independent coder approved by the researcher's supervisor was used. To facilitate the trustworthiness of the study, the principles of Lincoln and Guba (1985:290) were employed by the researcher. As the researcher is not experienced in conducting focus group interviews and due to the sensitivity of the topic, the researcher employed a professional facilitator. To ensure the quality of the research, the researcher adhered to the ethical standards set by the Democratic Nursing Organisation of South Africa (1998) in this regard.

\section{Results}

Six themes emerged from the identified problems and they are related to communication, inadequate staff competence, cost saving versus quality care, procedurat complexity, perceived loss of power by doctors and patients and account payment. Problems related to communication were grouped into information flow and poor relationship between the private hospitals and managed care organisations. Managed care organisations $(n=3)$, private hospital $(n=9)$ and general medical practitioners $(n=4)$ are experiencing difficulties obtaining information from each other to review the conditions of patients and for the managed care organisations to au 


\begin{tabular}{|c|c|c|}
\hline Hospitals & Managed care organisation & Doctors \\
\hline $\begin{array}{l}\text { a) Doctors refuse to give information } \\
\text { required by managed care organisa- } \\
\text { tions to hospital case managers. } \\
\text { b) Doctors refuse to write motivation } \\
\text { letters required by managed care or- } \\
\text { ganisations to do updates. } \\
\text { c) Patients come to hospitals for ad- } \\
\text { mission not knowing what they are } \\
\text { being admitted for. } \\
\text { d) Patients come to the hospitals with- } \\
\text { out authorisaion numbers. }\end{array}$ & $\begin{array}{l}\text { a) Doctors refuse to give necessary } \\
\text { information to managed care case } \\
\text { managers to do updates. } \\
\text { b) Doctors and hospitals do not ac- } \\
\text { cept information on managed health- } \\
\text { care because of their resistance to } \\
\text { change. } \\
\text { c) Doctors refuse to talk to managed } \\
\text { care case managers because they are } \\
\text { nurses and prefer to talk to doctors. } \\
\text { d) Patients phone managed care or- } \\
\text { ganisations for authorisation numbers } \\
\text { without necessary information to grant } \\
\text { such authorisation. } \\
\text { e) Doctors sometimes provide insuffi- } \\
\text { cient or incorrect information regard- } \\
\text { ing the diagnosis or treatment of the } \\
\text { patient. } \\
\text { f) When phoning the hospitals, there } \\
\text { is always insufficient information for } \\
\text { managed care case managers to do } \\
\text { updates. }\end{array}$ & $\begin{array}{l}\text { a) Lack of information from managed } \\
\text { care organisations to the patients on } \\
\text { managed healthcare. } \\
\text { b) There is no information flow from } \\
\text { managed care organisations to serv- } \\
\text { ice providers }\end{array}$ \\
\hline
\end{tabular}

thorise payment of services provided to patients, who are their members (see tables one and two).

Doctors refuse to provide necessary information required by managed care organisations to hospital case managers to authorise payment of services provided to patients because they feel like they are being checked upon by medically unqualified people. HASA (1999:100) confirms this situation: "Why should a nursing sister be allowed to challenge the opinion of an orthopedic surgeon that that an operation is not necessary? Should someone who is not medically qualified have the power to decide that the patient has stay enough in hospital?"

As a result, managed care case managers refuse to update the accounts or authorise services provided. Two participants $(n=2)$ confirmed the problem: "You know, doctors don't want to give us information required by these managed care people, and in this case, managed care people don't want to update our accounts.... when they don't get this information..."; "This is frustrating, doctors feel like they are being checked upon by these people who are not medically trained". Managed care case managers $(n=3)$ also share this problem with the private hospitals in that doctors refuse to give them necessary informa- tion to update the conditions of patients as they perceive managed healthcare as being there for managed care organisations to make money at their expense: " The whole myth that managed healthcare is there to save money and make profit you know, makes doctors to refuse to give us necessary information to update conditions of patients...".

Managed care organisations do not educate their members on the requirements of managed healthcare. As a result patients go to hospitals without knowing what they are getting admitted for, and without authorisation numbers. Hospital case managers $(n=3)$ experience frustration as they find themselves in situations where they phone doctors for patients' diagnoses and managed care organisations concerned for authorisation numbers: "Managed care organisations don't even inform their members on the expectations of managed care.You find patients coming to hospitals without knowing what they are getting admitted for, and without authorisation numbers". This problem is also experienced by general medical practitioners $(n=4)$. As a result they find themselves in a situation of educating patients on the requirements of their managed care organisations rather than treating them: "The most serious problem is that they are busy dictating to us, but they are failing to educate their members whom they are claiming to protect..., that is why members themselves don't know what their managed care organisations expect of them, and I think this is a serious problem, because we as doctors are finding ourselves in a situation whereby we educate patients on the rules of their medical aids and this is irritating". Managed care organisations are experiencing a problem of the hospitals and doctors not accepting information on managed healthcare because of their resistance to change, as confirmed by the following quotation: "You can give information and education to people, but if they are not willing, if they shut to it..., I think there are doctors and hospitals that understand it, but are not accepting it because of... and their resistance to change".

Doctors provide insufficient or incorrect information about the conditions and treatment of patients to managed care case managers, hoping that treatment and conditions that are normally not covered will be paid: “...I mean giving us insufficient information, this is frustrating and they also give us incorrect information.... when you receive the account, the information is not the same as what he told you when he phone, they think that this will make us pay for what we normally don't pay for". HASA 
Table 2: Summary of problems as experienced by the different role players

\begin{tabular}{|l|c|c|c|}
\hline Problem & Hospitals & $\begin{array}{c}\text { Managed care } \\
\text { organisations }\end{array}$ & Doctors \\
\hline $\begin{array}{l}\text { Communication } \quad \text { Information } \\
\text { Poor relationship }\end{array}$ & $\mathrm{x}$ & $\mathrm{x}$ & $\mathrm{x}$ \\
$\mathrm{x}$ & $\mathrm{x}$ & $\mathrm{x}$ & $\mathrm{X}$ \\
\hline Staff competence & $\mathrm{X}$ & $\mathrm{X}$ & \\
\hline Cost saving versus quality \\
care
\end{tabular}

${ }_{1} X$ indicates the response group

(1999:27) confirms these problems and emphasised that the information needed by managed care decisions is, in most cases, no known to the patient, the relative and other team members; there is no understanding among the medical aid beneficiaries as to how managed care works and how to access care within that system. The lack of education in managed healthcare is supported by Gallaway and Lee (1997:23), T.W.I.G Inform (1998:12). Table one summarises the information related problems as experienced by different role players.

Building meaningful relationships was experienced as a problem between the private hospitals and managed care organisations because of the lack of specific reliable contacts. Three participants emphasised the problem and the following direct quotations articulated the problem: "Another problem, another problem is making contacts with necessary people at the managed care organisations"; You also find that there are no reliable people to talk to for updates, because they still don't have case managers you know...".

Another source of the problems is the limited or no medical knowledge to certain people doing updates from other role players. All role players are experiencing a problem of talking to people with limited or no medical knowledge from the other two, which makes it difficult to update conditions of patients. This problem was emphasised by eight participants and the following selected direct quotations were used to emphasise the problem: "People that we talk to on the other side of medical aids don't have necessary medical knowledge...trying to explain to them the condition of the patient is oh...difficult, because the only thing that guide them are protocols"; "What about talking to the hospital confirmation clerks with those hospitals who don't have case managers?, it is difficult because they are not medically trained..."; "And eh...and also dictating to us as to how to operate, how to treat the patient, eh.. meanwhile these dictators don't even have medical knowledge, or in most cases, with managed care organisations are nurses, who come with unethical decisions... and not having an idea of the implications of their decisions".

Procedures followed by managed care organisations are perceived by private hospitals as rigid, and they are often not known to them, leading to frustration and resentment. This appears to be a manifestation of lack of standardisation in managed care industry. There is a strict adherence to protocols by managed care organisations without due consideration: "From the managed care organisations' side, protocols are a problem, protocols as opposed to what we do at the hospital"; "Rules differ from managed care organisation to managed care organisation you know, it is so amazing because eh..eh..they all expect us to adhere to all these rules".

Role players are experiencing a dilemma in balancing the provision of quality care, on the one hand, with the cost of providing the care to the member: "Another thing is that medical aids are desperate to save cost, rather than the quality of care provided to members, yet they are claiming to protect these members"; "...When you are in managed healthcare, you must prove your existance that you make a difference in an
organisation...We are forced into a situation where we have to say we have saved so much to prove our existance"; "Another problem is the preauthorisation, we still have... a lot of preauthorisation requests when patients are being at the door being admitted... and in that way, you cannot make an impact on the saving".

General medical practitioners feel that managed care organisations are taking over their power to decide on how and where the patient must be treated, and even dictating to patients as to who and where they must be treated: "I think these managed care organisations want to employ us and be in control of us, desperate to make profit...". This concern is supported by HASA (1999:49) : How can a protocol distinguish whether a headache needs an aspirin or whether it is related to a tumor?"

Doctor's accounts are paid late or not paid at all. Managed care organisations demand quality of care to their members from the service providers, yet they don't want to pay for it: "Accounts are not paid in time or not paid at all, this is frustrating as they expect us to provide quality care, yet they don't want to pay for this quality that they demand from us"; "I think they try and look for a good reason for not paying the account, and if they pay, they do not pay in time".

International data triangulation indicates that the USA also experienced these problems in the implementation of man. aged healthcare by different role players. Not all role players experienced all problems, similarly, some problems were experienced by more than one role player. Table two provides a summary of the problems as experienced by different role players. 
Solutions are suggested by the participants themselves to counteract the experienced problems. Suggested solutions revolved around the standardisation of methods, procedures and systems followed in the managed healthcare industry, involvement of all role players in the formulation of the guidelines and standards, role players education on managed healthcare, recruitment and employment of medically qualified staff to be case managers by both the private hospitals and managed care organizations, employment of doctors by managed care organizations to make medical decisions and member education. These solutions were seen by role players as important for enhancing positive working relationship in the delivery of healthcare within the managed healthcare context in Gauteng.

\section{Conclusions And Recommendations}

The following conclusions are made: Problems experienced by the role players within the managed healthcare context in Gauteng were related to communication, staff competence, cost saving versus quality care, procedural complexity, perceived loss of power by doctors and patients and accounts payment.

- Communication problems relate to difficulty in the flow of information among the role players and difficulty in building meaningful relationship.

- All role players experience a problem of talking to people with limited or no medical knowledge from the other two.

- Managed care case managers need to prove their existance to their organisations by saving costs and as a result, the care provided to patients is compromised.

- Managed care organisations expect other role players to work according to their procedures rather than what is best for the patients.

- Managed care organisations dictate to other role players.

- Managed care organisations don't pay the doctors' accounts in time or they don't pay them at all.

- All role players suggested solutions to counteract the problems and solutions revolved around the standardisation of managed healthcare methods across managed health care industry, role player involvement in standards and guidelines formulation, role player empowerment and member education and formation of independent practitioners' association by doctors.

From the identified problems and suggested solutions, the following recommendations were made:
- Replication studies to be conducted in other provinces of South Africa.

- Problems experienced by the patients within the context of managed healthcare in Gauteng to be explored and described.

- Ethical standards to be formulated for the role players in the provision of healthcare.

- The roles of different role players in the provision of healthcare within the context of managed healthcare to be clarified.

- A clear job description of a case manager to be formulated.

- A clear inservice training programme for a case managers to be formulated.

\section{Limitations}

The sensitive nature of the topic could have influenced the process and the content of data collection. Participants were interviewed in different venues, which could have influenced the nature of the results. The professional facilitator was not probing enough information from the participants, which could have influenced the nature of the results.

\section{Concluding Remarks}

Problems experienced by the role players within the context of managed healthcare have a negative effect on their relationship, which in turn affect the quality of care provided to patients. As managed healthcare is a new concept in South Africa, little was known about the problems. Exploration and description of such problems lead to their better understanding and the research results were used as guidelines for the formulation of the strategy to enhance positive working relationship among the role players within the managed healthcare context in Gauteng.

\section{Acknowledgement}

All participants are hereby acknowledged and thanked for their contributions, and regardless of the sensitivity of the topic, were willing to participate.

\section{References}

BARR, D. 1997. Structuring Managed Care Delivery Systems to increase primary care quality; Abstract Book Association for Services Research, 14: 3-4.

DE VOS, A.S. (Ed.) 1998. Research at grass roots: a primer for the caring professions Pretoria: Van Schaik.

DENOSA. 1998. Ethical standards for nursing research. Pretoria: DENOSA.

GALLAWAY, S.J., \& LEE, B. 1997. Parish nursing: opportunities in community health. Journal Article of home care provided, 2(5):244-249.

HASA. 1998. Private Health Care. Seventh annual publication. Greenside: Wilbury \& Claymore.

HASA. 1999. Private Healthcare. Eighth annual pulication. Greenside: Wilbury \& Claymore.

KRUEGER, R.A. 1988. Focus groups: a practical guide for applied research. Newsbury Park: Sage publications.

LINCOLN, Y.S., \& GUBA, E. 1985. Naturalistic inquiry. London: Sage.

TESCH, R. 1990. Qualitative research: analysis, types of software tools. New York: Palmer Press.

T.W.I.G. INFORM. 1998. Managed care in South Africa today. (Unpublished study).

VELIOTES, G., MAGENNIS, R., \& BROWN, M. 1993. Introduction of managed healthcare in South Africa. Pretoria: State Press. 